\title{
CTLA-4 polymorphisms and predisposition to digestive system malignancies: a meta- analysis of 31 published studies
}

\author{
Jien Li', Wenping Wang ${ }^{2}$, Yanyan Sun ${ }^{1}$ and Yeduo Zhu ${ }^{3^{*}}$
}

\begin{abstract}
Background: The results of genetic association studies regarding cytotoxic T lymphocyte-associated antigen 4 (CTLA-4) polymorphisms and digestive system malignancies were controversial. The authors designed this metaanalysis to more precisely estimate relationships between CTLA-4 polymorphisms and digestive system malignancies by pooling the results of related studies.

Methods: The authors searched PubMed, Embase, Web of Science, and CNKI for eligible studies. Thirty-one eligible studies were pooled analyzed in this meta-analysis.

Results: The pooled meta-analysis results showed that genetic distributions of rs231775, rs4553808, and rs733618 polymorphisms among patients with digestive system malignancies and controls differed significantly. Moreover, genotypic distribution differences were also observed for rs231775 polymorphism among patients with colorectal cancer/pancreatic cancer and controls, for rs4553808 and rs5742909 polymorphisms among patients with gastric cancer and controls, for rs3087243 polymorphism among patients with liver cancer and controls, and for rs 733618 polymorphism among patients with colorectal cancer and controls in pooled meta-analyses.

Conclusions: This meta-analysis suggested that rs231775 polymorphism was associated with predisposition to colorectal cancer and pancreatic cancer, rs4553808 and rs5742909 polymorphisms were associated with predisposition to gastric cancer, rs3087243 polymorphism was associated with predisposition to liver cancer, and rs733618 polymorphism was associated with predisposition to colorectal cancer.
\end{abstract}

Keywords: Cytotoxic T lymphocyte-associated antigen 4 (CTLA-4), Polymorphisms, Digestive system malignancies, Meta-analysis

\section{Background}

Digestive system malignancies such as liver cancer, gastric cancer, and colorectal cancer are leading types of cancer among both males and females [1, 2]. Although their definite pathogenesis mechanisms are still unclear, accumulating evidence suggests that genetic architecture plays vital roles in their development. Firstly, the incidences of digestive system malignancies have been found to be higher in subjects with positive family history in first-degree relatives [3-5], and genetic background is probably one of the reasons behind this phenomenon.

\footnotetext{
*Correspondence: dauozhuyes@163.com

${ }^{3}$ Department of Gastroenterology, Zhuji Affiliated Hospital of Shaoxing

University, Zhuji 311800, Zhejiang, China

Full list of author information is available at the end of the article
}

Secondly, previous genetic association studies have also detected numerous susceptible genetic loci of digestive system malignancies in different populations [6-8]. However, the pathogenesis mechanisms of digestive system malignancies are very complicated, and genetic factors that contribute to the development of digestive system malignancies still require intensive explorations.

Cytotoxic T lymphocyte-associated antigen 4 (CTLA4) serves as a negative regulator of immune responses and is essential for modulating anti-tumor immune responses $[9,10]$. So, if a polymorphism is of potential functional significance and can impact the gene expression or protein structure of CTLA-4, it is likely that this polymorphism might also influence predisposition to

(C) The Author(s). 2020 Open Access This article is distributed under the terms of the Creative Commons Attribution 4.0 International License (http://creativecommons.org/licenses/by/4.0/), which permits unrestricted use, distribution, and reproduction in any medium, provided you give appropriate credit to the original author(s) and the source, provide a link to the Creative Commons license, and indicate if changes were made. The Creative Commons Public Domain Dedication waiver (http://creativecommons.org/publicdomain/zero/1.0/) applies to the data made available in this article, unless otherwise stated. 
many types of malignant diseases including digestive system malignancies.

In the last two decades, investigators across the world have extensively explored the relationship between CTLA-4 polymorphisms and digestive system malignancies, yet the relationships between CTLA-4 polymorphisms and digestive system malignancies are still controversial and ambiguous. Thus, the authors designed this meta-analysis to get a more statistically reliable conclusion regarding the relationships between CTLA-4 polymorphisms and digestive system malignancies by pooling the results of related studies.

\section{Methods}

The PRISMA guideline was followed by the authors when conducting this meta-analysis [11].

\section{Literature search and inclusion criteria}

Literature searching of PubMed, Web of Science, Embase, and CNKI was performed by the authors using the following terms: (Cytotoxic T lymphocyte antigen-4 or CTLA-4) and (polymorphism or variant or variation or mutation or SNP or genome-wide association study or genetic association study or genotype or allele) and (colorectal or colon or rectal or pancreatic or pancreas or esophageal or esophagus or gastric or stomach or liver or hepatic) and (cancer or tumor or carcinoma or neoplasm or malignancy). The authors also checked the references of retrieved articles for additional related studies.

Eligible studies must meet all of the three inclusion criteria: (I) formally published case-control studies evaluating relationships between CTLA-4 polymorphisms and digestive system malignancies, (II) provide genotypic distributions of CTLA-4 polymorphisms in patients with digestive system malignancies and controls, and (III) the full manuscript is available in English or Chinese. Articles were excluded if at least one of the following three conditions was fulfilled: (I) studies not concerning CTLA-4 polymorphisms and digestive system malignancies, (II) reviews or expert comments, and (III) case series that only involved patients with digestive system malignancies. When duplicate reports were observed during literature searching, only the most complete one was included for pooled analyses.

\section{Data extraction and quality assessment}

We extracted the following items from eligible studies: (I) surname of the first author, (II) year of online publication, (III) country and ethnicity of involved subjects, (IV) number of patients and controls in each study, and (V) genotypic distributions of CTLA-4 polymorphisms in patients and control subjects. We also calculated the $p$ values of Hardy-Weinberg equilibrium (HWE) based on genotypic distributions of CTLA-4 polymorphisms.
The authors used the Newcastle-Ottawa scale (NOS) to assess the quality of included studies [12]. The score range of NOS is from zero to nine, and the methodology quality of a study is considered to be good if it can get a score of more than seven.

Data extraction and quality assessment of eligible studies were performed by two authors separately. We would write to the corresponding authors of eligible studies for additional data if we fail to extract necessary information from included studies.

\section{Statistical analyses}

The authors used Review Manager to pool the results of eligible studies. The authors used $Z$ test to evaluate the relationships between CTLA-4 polymorphisms and predisposition to digestive system malignancies. The authors set the statistical significant threshold at 0.05 . We compared genetic distributions of CTLA-4 polymorphisms among cases and controls in dominant, recessive, over-dominant, and allele models; the dominant genetic model is defined as $M / M$ vs. $M / m+m / m$, recessive genetic model is defined as $\mathrm{m} / \mathrm{m}$ vs. $\mathrm{M} / \mathrm{M}+\mathrm{M} / \mathrm{m}$, over-dominant genetic model is defined as $M / m$ vs. $M / M+m / m$, and the allele genetic model is defined as $\mathrm{M}$ vs. $\mathrm{m}$. The authors used $I^{2}$ statistics to estimate heterogeneity. The authors used the DerSimonian-Laird method to pool the results if $I^{2}$ is larger than 50\%. Otherwise, the authors used the MantelHaenszel method to pool the results. The authors also conducted subgroup analyses by type of diseases. The authors examined the stabilities of pooled meta-analysis results by omitting one study each time and pooling the results of the other studies. The authors examined publication biases by using funnel plots.

\section{Results}

\section{Characteristics of included studies}

One hundred and thirty-two articles were retrieved by the authors through our literature searching strategy. The authors assessed 54 articles for eligibility after omitting unrelated and repeated reports. Twenty reviews were further excluded by the authors, and another three articles were also excluded by the authors due to the lack of crucial data. Totally, 31 studies were finally pooled in our meta-analyses (Fig. 1). Extracted data of eligible studies were summarized in Table 1.

\section{Meta-analysis results of CTLA-4 polymorphisms and digestive system malignancies}

Twenty-eight studies were eligible for estimation of relationship between rs 231775 polymorphism and digestive system malignancies, three studies were eligible for estimation of relationship between rs4553808 polymorphism and digestive system malignancies, six studies were eligible for estimation of relationship between rs5742909 


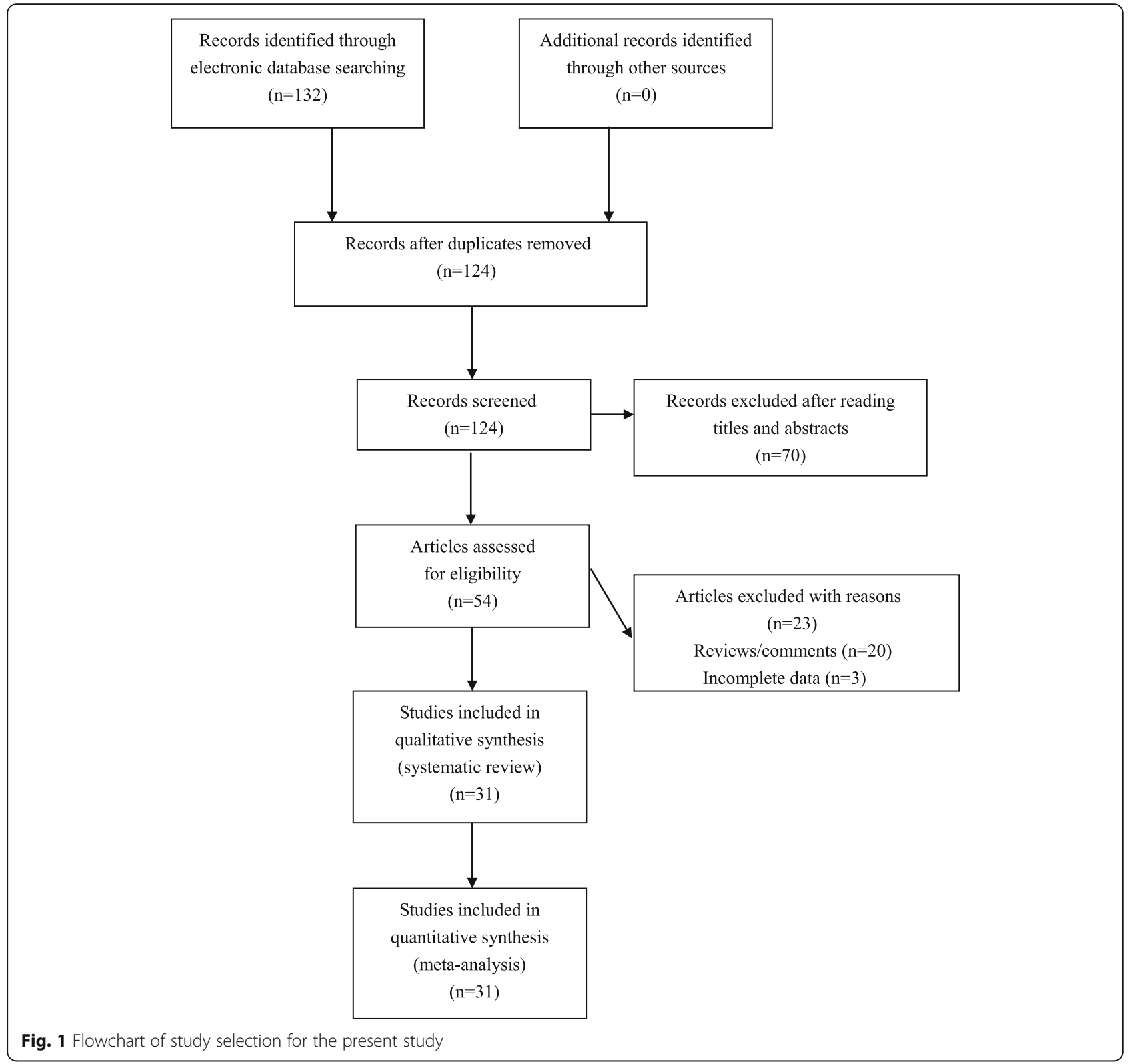

polymorphism and digestive system malignancies, eight studies were eligible for estimation of relationship between rs3087243 polymorphism and digestive system malignancies, eight studies were eligible for estimation of relationship between rs733618 polymorphism and digestive system malignancies, and four studies were eligible for estimation of relationship between rs16840252 polymorphism and digestive system malignancies. CTLA-4 rs231775 (over-dominant comparison: $\mathrm{OR}=1.06, p=0.03$ ), rs4553808 (dominant comparison: $\mathrm{OR}=0.77, p=0.04$; recessive comparison: $\mathrm{OR}=0.52, p=0.003$; over-dominant comparison: $\mathrm{OR}=1.73, p<0.0001$ ), and rs733618 (overdominant comparison: $\mathrm{OR}=1.27, p=0.04$ ) polymorphisms were found to be significantly associated with digestive system malignancies in overall pooled metaanalyses. We also obtained positive findings for rs 231775 polymorphism in colorectal cancer (recessive and overdominant comparisons) and pancreatic cancer (dominant, recessive, over-dominant, and allele comparisons) subgroups, for rs4553808 polymorphism in gastric cancer (recessive and over-dominant comparisons) subgroup, for rs5742909 polymorphism in gastric cancer (dominant and allele comparisons) subgroup, for rs3087243 polymorphism in liver cancer (dominant, recessive, and allele comparisons) subgroup, and for rs733618 polymorphism in colorectal cancer (allele comparison) subgroup. Nevertheless, no any positive results were observed for rs16840252 polymorphism in pooled meta-analyses (see Table 2 ). 
Table 1 The characteristics of included studies for this meta-analysis

\begin{tabular}{|c|c|c|c|c|c|c|c|c|}
\hline \multirow[t]{2}{*}{ First author, year } & \multirow[t]{2}{*}{ Country } & \multirow[t]{2}{*}{ Ethnicity } & \multirow[t]{2}{*}{ Type of disease } & \multirow{2}{*}{$\begin{array}{l}\text { Sample } \\
\text { size }\end{array}$} & \multicolumn{2}{|c|}{ Genotypes (wtwt/wtmt/mtmt) } & \multirow{2}{*}{$\begin{array}{l}P \text { value } \\
\text { for } \\
\text { HWE }\end{array}$} & \multirow{2}{*}{$\begin{array}{l}\text { NOS } \\
\text { scor }\end{array}$} \\
\hline & & & & & Cases & Controls & & \\
\hline \multicolumn{9}{|l|}{$\mathrm{rs} 231775 \mathrm{~A} / \mathrm{G}$} \\
\hline Cai $2011^{*}$ & China & East Asian & Esophageal cancer & $125 / 250$ & $30 / 68 / 27$ & 70/133/47 & 0.248 & 8 \\
\hline Cheng 2006* & Taiwan & East Asian & Gastric cancer & $62 / 250$ & $34 / 26 / 2$ & $119 / 102 / 29$ & 0.323 & 7 \\
\hline Cheng $2011^{*}$ & China & East Asian & Esophageal cancer & $205 / 205$ & $54 / 105 / 46$ & $90 / 79 / 36$ & 0.013 & 7 \\
\hline Cozar 2007* & Spain & Caucasian & Colorectal cancer & $96 / 176$ & $46 / 44 / 6$ & $78 / 77 / 21$ & 0.766 & 8 \\
\hline Cui $2012^{*}$ & China & East Asian & Colorectal cancer & $128 / 205$ & $73 / 46 / 9$ & $84 / 68 / 53$ & $<0.001$ & 7 \\
\hline Cui $2016^{*}$ & China & East Asian & Liver cancer & $96 / 205$ & $52 / 37 / 7$ & $64 / 96 / 45$ & 0.429 & 7 \\
\hline Dilmec 2008* & Turkey & Mixed & Colorectal cancer & $56 / 162$ & $36 / 19 / 1$ & 108/43/11 & 0.028 & 7 \\
\hline Fan $2012^{*}$ & China & East Asian & Colorectal cancer & $291 / 352$ & $123 / 146 / 22$ & $170 / 138 / 44$ & 0.059 & 8 \\
\hline Ge $2015^{*}$ & China & East Asian & Colorectal cancer & $572 / 626$ & 296/242/34 & $292 / 284 / 50$ & 0.094 & 7 \\
\hline Gu 2010* & China & East Asian & Liver cancer & $367 / 407$ & $150 / 166 / 51$ & 183/179/45 & 0.902 & 8 \\
\hline Hadinia $2007^{*}$ & Iran & Mixed & Colorectal cancer & $105 / 190$ & $52 / 47 / 6$ & $117 / 59 / 14$ & 0.097 & 8 \\
\hline Hadinia $2007^{*}$ & Iran & Mixed & Gastric cancer & $46 / 190$ & 27/13/6 & $117 / 59 / 14$ & 0.097 & 8 \\
\hline Hou $2010^{*}$ & China & East Asian & Gastric cancer & $205 / 262$ & $94 / 70 / 41$ & $107 / 100 / 55$ & $<0.001$ & 7 \\
\hline $\mathrm{Hu} 2010^{*}$ & China & East Asian & Liver cancer & $853 / 854$ & $367 / 380 / 106$ & $399 / 376 / 79$ & 0.476 & 8 \\
\hline Lang 2012* & China & East Asian & Pancreatic cancer & $602 / 651$ & 208/312/82 & $263 / 326 / 62$ & 0.006 & 8 \\
\hline Li 2011* & China & East Asian & Colorectal cancer & $248 / 380$ & $120 / 120 / 8$ & $171 / 167 / 42$ & 0.898 & 8 \\
\hline Liu 2015* & China & East Asian & Liver cancer & $80 / 78$ & $29 / 36 / 15$ & $38 / 33 / 7$ & 0.966 & 7 \\
\hline Liu $2015^{*}$ & China & East Asian & Esophageal cancer & $629 / 686$ & $307 / 254 / 43$ & $310 / 296 / 58$ & 0.284 & 7 \\
\hline Liu 2019* & China & East Asian & Gastric cancer & $487 / 1470$ & $228 / 215 / 44$ & $698 / 631 / 141$ & 0.926 & 7 \\
\hline Mahajan 2008* & Poland & Caucasian & Gastric cancer & $301 / 411$ & $89 / 153 / 59$ & $152 / 189 / 70$ & 0.393 & 7 \\
\hline Qi 2010* & China & East Asian & Colorectal cancer & $124 / 407$ & $60 / 60 / 4$ & 183/179/45 & 0.902 & 8 \\
\hline Solerio $2005^{*}$ & Italy & Caucasian & Colorectal cancer & $132 / 238$ & $76 / 43 / 13$ & 128/91/19 & 0.618 & 8 \\
\hline Sun $2008^{*}$ & China & East Asian & Gastric cancer & $530 / 530$ & $235 / 235 / 60$ & 282/209/39 & 0.974 & 8 \\
\hline Sun $2008^{*}$ & China & East Asian & Esophageal cancer & $629 / 686$ & $307 / 254 / 43$ & $310 / 290 / 58$ & 0.398 & 8 \\
\hline Tang 2016* & China & East Asian & Gastric cancer & $330 / 590$ & 155/153/22 & $278 / 264 / 48$ & 0.179 & 8 \\
\hline Wang $2015^{*}$ & China & East Asian & Colorectal cancer & $311 / 389$ & $121 / 147 / 43$ & $141 / 147 / 101$ & $<0.001$ & 7 \\
\hline Yang 2012* & China & East Asian & Pancreatic cancer & $368 / 926$ & 140/178/50 & $482 / 374 / 70$ & 0.828 & 8 \\
\hline Yang 2015* & China & East Asian & Colorectal cancer & $240 / 147$ & 195/39/6 & $102 / 40 / 5$ & 0.662 & 7 \\
\hline Yang 2019* & China & East Asian & Liver cancer & $575 / 920$ & 290/221/64 & $444 / 389 / 87$ & 0.893 & 8 \\
\hline Yuan 2012* & China & East Asian & Gastric cancer & $118 / 96$ & $65 / 45 / 8$ & $30 / 45 / 21$ & 0.595 & 7 \\
\hline Zou 2018* & China & East Asian & Colorectal cancer & 979/1299 & $417 / 443 / 119$ & $621 / 563 / 115$ & 0.430 & 8 \\
\hline \multicolumn{9}{|l|}{ rs4553808 A/G } \\
\hline Cui 2016* & China & East Asian & Liver cancer & $96 / 205$ & $33 / 56 / 7$ & 79/96/30 & 0.924 & 7 \\
\hline Hadinia $2007^{*}$ & Iran & Mixed & Colorectal cancer & 109/188 & $74 / 33 / 2$ & $145 / 36 / 7$ & 0.02 & 7 \\
\hline Hadinia $2007^{*}$ & Iran & Mixed & Gastric cancer & $46 / 188$ & $37 / 9 / 0$ & $145 / 36 / 7$ & 0.02 & 7 \\
\hline Hou $2010^{*}$ & China & East Asian & Gastric cancer & $205 / 262$ & $112 / 71 / 22$ & $163 / 54 / 45$ & $<0.001$ & 7 \\
\hline \multicolumn{9}{|l|}{ rs5742909 C/T } \\
\hline Cheng 2006* & Taiwan & East Asian & Gastric cancer & $62 / 250$ & $59 / 3 / 0$ & 209/40/1 & 0.323 & 7 \\
\hline Dilmec 2008* & Turkey & Mixed & Colorectal cancer & $56 / 162$ & $48 / 8 / 0$ & 149/12/1 & 0.185 & 7 \\
\hline Hadinia $2007^{*}$ & Iran & Mixed & Gastric cancer & $46 / 187$ & $38 / 8 / 0$ & $159 / 24 / 4$ & 0.014 & 8 \\
\hline Hadinia 2007* & Iran & Mixed & Colorectal cancer & 108/187 & $91 / 16 / 1$ & $159 / 24 / 4$ & 0.013 & 7 \\
\hline Li 2009* & China & East Asian & Gastric cancer & $236 / 121$ & $206 / 27 / 3$ & $99 / 17 / 5$ & 0.001 & 7 \\
\hline
\end{tabular}


Table 1 The characteristics of included studies for this meta-analysis (Continued)

\begin{tabular}{|c|c|c|c|c|c|c|c|c|}
\hline \multirow[t]{2}{*}{ First author, year } & \multirow[t]{2}{*}{ Country } & \multirow[t]{2}{*}{ Ethnicity } & \multirow[t]{2}{*}{ Type of disease } & \multirow{2}{*}{$\begin{array}{l}\text { Sample } \\
\text { size }\end{array}$} & \multicolumn{2}{|c|}{ Genotypes (wtwt/wtmt/mtmt) } & \multirow{2}{*}{$\begin{array}{l}P \text { value } \\
\text { for } \\
\text { HWE }\end{array}$} & \multirow{2}{*}{$\begin{array}{l}\text { NOS } \\
\text { score }\end{array}$} \\
\hline & & & & & Cases & Controls & & \\
\hline Wang 2019* & China & East Asian & Liver cancer & $554 / 612$ & $360 / 170 / 24$ & $466 / 134 / 12$ & 0.517 & 8 \\
\hline Yang 2015* & China & East Asian & Colorectal cancer & $240 / 147$ & $150 / 82 / 8$ & $94 / 48 / 5$ & 0.707 & 7 \\
\hline \multicolumn{9}{|l|}{ rs3087243 CT60AG } \\
\hline Cheng 2006* & Taiwan & East Asian & Gastric cancer & $62 / 250$ & $39 / 20 / 3$ & $154 / 79 / 17$ & 0.126 & 7 \\
\hline Cozar 2007* & Spain & Caucasian & Colorectal cancer & $95 / 175$ & 20/56/19 & $40 / 88 / 47$ & 0.923 & 7 \\
\hline Ge $2015^{*}$ & China & East Asian & Colorectal cancer & $1699 / 627$ & $1258 / 425 / 16$ & $413 / 198 / 16$ & 0.174 & 8 \\
\hline Liu 2019* & China & East Asian & Gastric cancer & $487 / 1472$ & $302 / 172 / 13$ & $958 / 462 / 52$ & 0.686 & 8 \\
\hline Tang 2016* & China & East Asian & Gastric cancer & $316 / 580$ & $213 / 98 / 5$ & $382 / 182 / 16$ & 0.302 & 8 \\
\hline Wang 2019* & China & East Asian & Liver cancer & $554 / 612$ & 200/238/116 & 240/274/98 & 0.185 & 8 \\
\hline Yang 2019* & China & East Asian & Liver cancer & $575 / 921$ & $325 / 221 / 29$ & $609 / 282 / 30$ & 0.703 & 8 \\
\hline Zou 2018* & China & East Asian & Colorectal cancer & $980 / 1300$ & 637/296/47 & $850 / 408 / 42$ & 0.410 & 8 \\
\hline \multicolumn{9}{|l|}{ rs733618 T/C } \\
\hline Cui 2016* & China & East Asian & Liver cancer & $96 / 205$ & $72 / 24 / 0$ & $181 / 24 / 0$ & 0.373 & 7 \\
\hline Hadinia $2007^{*}$ & Iran & Mixed & Colorectal cancer & 109/189 & $97 / 12 / 0$ & $165 / 24 / 0$ & 0.351 & 7 \\
\hline Hadinia $2007^{*}$ & Iran & Mixed & Gastric cancer & $83 / 189$ & $42 / 41 / 0$ & $165 / 24 / 0$ & 0.351 & 7 \\
\hline Hou $2010^{*}$ & China & East Asian & Gastric cancer & $205 / 262$ & 75/111/19 & $93 / 139 / 30$ & 0.041 & 7 \\
\hline Liu 2019* & China & East Asian & Gastric cancer & $487 / 1472$ & $168 / 242 / 77$ & $525 / 685 / 262$ & 0.139 & 8 \\
\hline Tang 2014* & China & East Asian & Esophageal cancer & $611 / 657$ & 210/300/101 & 228/314/115 & 0.700 & 8 \\
\hline Tang 2016* & China & East Asian & Gastric cancer & $320 / 586$ & 102/163/55 & 198/282/106 & 0.749 & 8 \\
\hline Yang 2019* & China & East Asian & Liver cancer & $575 / 921$ & 217/268/90 & $320 / 432 / 169$ & 0.275 & 8 \\
\hline Zou 2018* & China & East Asian & Colorectal cancer & $980 / 1300$ & $346 / 464 / 170$ & $458 / 613 / 229$ & 0.335 & 8 \\
\hline \multicolumn{9}{|l|}{ rs16840252 C/T } \\
\hline Liu 2019* & China & East Asian & Gastric cancer & $492 / 1472$ & $381 / 94 / 7$ & $1130 / 329 / 13$ & 0.039 & 8 \\
\hline Tang 2016* & China & East Asian & Gastric cancer & $317 / 603$ & $235 / 78 / 4$ & 460/130/13 & 0.293 & 8 \\
\hline Yang 2019* & China & East Asian & Liver cancer & $575 / 921$ & $477 / 93 / 5$ & $707 / 205 / 9$ & 0.164 & 8 \\
\hline Zou 2018* & China & East Asian & Colorectal cancer & $980 / 1300$ & 742/223/15 & 1006/283/11 & 0.065 & 8 \\
\hline
\end{tabular}

Abbreviations: wt wild type, $m t$ mutant type, HWE Hardy-Weinberg equilibrium, NOS Newcastle-Ottawa scale, NA not available

*Full manuscript of all eligible studies can be accessed at https://osf.io

\section{Sensitivity analyses}

Stabilities of pooled meta-analysis results were examined by omitting one study each time and pooling the results of the other studies. The trends of associations remained unchanged in sensitivity analyses, indicating that our pooled meta-analysis results were statistically stable.

\section{Publication biases}

Publication biases were examined by funnel plots. Funnel plots were overall symmetrical, suggesting that our pooled meta-analysis results were not likely to be severely influenced by publication biases.

\section{Discussion}

CTLA-4 is expressed on activated T cells, and it negatively regulates $\mathrm{T}$ cell activation and proliferation. Previous studies have demonstrated that CTLA-4 modulates the duration and strength of $\mathrm{T}$ cell-mediated immune responses by competitive binding with co-stimulating B7 molecules and activating of FAS-dependent apoptosis of T cells $[9,10]$. Recently, abnormal expression of CTLA-4 gene has been documented in many types of cancers, and it might contribute to cancer initiation and progression [1315]. Considering that genetic polymorphisms might influence gene expression or even protein function, CTLA-4 gene polymorphisms have also been extensively explored with regard to their relationships with different types of malignant disorders. In this meta-analysis, we summarized potential relationships between CTLA-4 gene polymorphisms and digestive system malignancies by pooling the results of 31 related studies. Our pooled meta-analysis results demonstrated that rs231775 polymorphism was associated with predisposition to colorectal cancer and pancreatic cancer, rs4553808 and rs5742909 polymorphisms were associated 
Table 2 Meta-analysis results of this study

\begin{tabular}{|c|c|c|c|c|c|c|c|c|c|}
\hline \multirow[t]{3}{*}{ Variables } & \multirow[t]{3}{*}{ Sample size } & \multirow{2}{*}{\multicolumn{2}{|c|}{$\frac{\text { Dominant comparison }}{M / M \text { vs. } M / m+m / m}$}} & \multirow{2}{*}{\multicolumn{2}{|c|}{$\frac{\text { Recessive comparison }}{m / m \text { vs. } M / M+M / m}$}} & \multirow{2}{*}{\multicolumn{2}{|c|}{$\frac{\text { Over-dominant comparison }}{M / m \text { vs. } M / M+m / m}$}} & \multirow{2}{*}{\multicolumn{2}{|c|}{$\frac{\text { Allele comparison }}{\mathrm{M} \text { vs. } \mathrm{m}}$}} \\
\hline & & & & & & & & & \\
\hline & & $p$ value & OR $(95 \% \mathrm{Cl})$ & $p$ value & OR $(95 \% \mathrm{Cl})$ & $p$ value & OR $(95 \% \mathrm{Cl})$ & $p$ value & OR (95\%Cl) \\
\hline \multicolumn{10}{|l|}{ rs231775 } \\
\hline Overall & $9890 / 14,238$ & 0.91 & $0.99(0.89-1.11)$ & 0.13 & $0.86(0.71-1.05)$ & 0.03 & $1.06(1.00-1.12)$ & 0.22 & $1.06(0.96-1.17)$ \\
\hline Esophageal cancer & $1588 / 1827$ & 0.47 & $0.87(0.60-1.26)$ & 0.71 & $0.96(0.77-1.20)$ & 0.67 & $1.05(0.83-1.35)$ & 0.57 & $0.93(0.93-1.19)$ \\
\hline Gastric cancer & 2079/3799 & 0.84 & $1.02(0.81-1.29)$ & 0.65 & $0.92(0.66-1.30)$ & 0.27 & $1.07(0.95-1.19)$ & 0.16 & $1.14(0.95-1.37)$ \\
\hline Colorectal cancer & $3042 / 4424$ & 0.64 & $1.04(0.88-1.23)$ & 0.007 & $0.55(0.35-0.84)$ & 0.006 & $1.14(1.04-1.25)$ & 0.09 & $1.16(0.98-1.38)$ \\
\hline Liver cancer & $1971 / 2464$ & 0.82 & $1.04(0.76-1.41)$ & 0.57 & $1.13(0.75-1.70)$ & 0.41 & $0.95(0.84-1.07)$ & 0.98 & $1.00(0.77-1.31)$ \\
\hline Pancreatic cancer & $970 / 1577$ & $<0.0001$ & $0.67(0.57-0.79)$ & 0.0001 & $1.67(1.29-2.16)$ & 0.03 & $1.20(1.02-1.42)$ & $<0.0001$ & $0.73(0.64-0.82)$ \\
\hline \multicolumn{10}{|l|}{ rs4553808 } \\
\hline Overall & $456 / 843$ & 0.04 & $0.77(0.60-0.98)$ & 0.003 & $0.52(0.34-0.81)$ & $<0.0001$ & $1.73(1.34-2.24)$ & 0.87 & $0.98(0.81-1.19)$ \\
\hline Gastric cancer & $251 / 450$ & 0.20 & $0.80(0.57-1.12)$ & 0.03 & $0.56(0.33-0.95)$ & 0.002 & $1.77(1.23-2.55)$ & 0.86 & $1.02(0.78-1.34)$ \\
\hline \multicolumn{10}{|l|}{ rs5742909 } \\
\hline Overall & $1302 / 1666$ & 0.76 & $0.94(0.63-1.40)$ & 0.42 & $1.22(0.75-1.99)$ & 0.42 & $1.15(0.82-1.62)$ & 0.58 & $0.87(0.54-1.41)$ \\
\hline Gastric cancer & $344 / 558$ & 0.04 & $1.58(1.01-2.48)$ & 0.14 & $0.40(0.12-1.36)$ & 0.46 & $0.74(0.33-1.66)$ & 0.01 & $1.69(1.12-2.56)$ \\
\hline Colorectal cancer & $404 / 496$ & 0.45 & $0.88(0.63-1.23)$ & 0.66 & $0.81(0.32-2.08)$ & 0.33 & $1.19(0.84-1.68)$ & 0.15 & $0.58(0.28-1.21)$ \\
\hline \multicolumn{10}{|l|}{ rs3087243 } \\
\hline Overall & $4768 / 5937$ & 0.72 & $0.96(0.79-1.17)$ & 0.66 & $0.92(0.64-1.33)$ & 0.70 & $1.03(0.87-1.23)$ & 0.90 & $0.99(0.84-1.17)$ \\
\hline Gastric cancer & $865 / 2302$ & 0.53 & $0.95(0.81-1.12)$ & 0.14 & $0.69(0.43-1.13)$ & 0.23 & $1.11(0.94-1.31)$ & 0.95 & $1.00(0.86-1.15)$ \\
\hline Colorectal cancer & $2774 / 2102$ & 0.45 & $1.14(0.81-1.59)$ & 0.49 & $0.75(0.32-1.72)$ & 0.58 & $0.92(0.69-1.23)$ & 0.39 & $1.15(0.84-1.56)$ \\
\hline Liver cancer & $1129 / 1533$ & 0.04 & $0.76(0.58-0.99)$ & 0.006 & $1.43(1.11-1.85)$ & 0.51 & $1.15(0.76-1.73)$ & $<0.0001$ & $0.78(0.69-0.88)$ \\
\hline \multicolumn{10}{|l|}{ rs733618 } \\
\hline Overall & $3466 / 5781$ & 0.10 & $0.81(0.64-1.04)$ & 0.12 & $0.91(0.80-1.02)$ & 0.04 & $1.27(1.01-1.59)$ & 0.16 & $0.88(0.74-1.05)$ \\
\hline Gastric cancer & $1095 / 2509$ & 0.13 & $0.65(0.37-1.14)$ & 0.23 & $0.88(0.72-1.08)$ & 0.08 & $1.60(0.95-2.70)$ & 0.18 & $0.77(0.52-1.13)$ \\
\hline Colorectal cancer & 1089/1489 & 0.89 & $1.01(0.85-1.20)$ & 0.87 & $0.98(0.79-1.22)$ & 0.99 & $1.00(0.85-1.18)$ & 0.05 & $0.89(0.79-1.00)$ \\
\hline Liver cancer & $671 / 1126$ & 0.50 & $0.70(0.25-1.96)$ & 0.18 & $0.83(0.62-1.09)$ & 0.38 & $1.50(0.60-3.73)$ & 0.51 & $0.73(0.29-1.85)$ \\
\hline \multicolumn{10}{|l|}{ rs16840252 } \\
\hline Overall & $2364 / 4296$ & 0.64 & $1.05(0.85-1.31)$ & 0.94 & $0.99(0.73-1.34)$ & 0.44 & $0.91(0.72-1.16)$ & 0.89 & $1.01(0.83-1.24)$ \\
\hline Gastric cancer & $809 / 2075$ & 0.84 & $0.98(0.81-1.19)$ & 0.19 & $1.22(0.90-1.66)$ & 0.89 & $0.97(0.68-1.40)$ & 0.39 & $0.93(0.78-1.10)$ \\
\hline
\end{tabular}

with predisposition to gastric cancer, rs3087243 polymorphism was associated with predisposition to liver cancer, and rs733618 polymorphism was associated with predisposition to colorectal cancer. The trends of associations remained unchanged in sensitivity analyses, suggesting that our pooled meta-analysis results were quite statistically stable.

A few points should be considered when interpreting our findings. First, previous experimental studies demonstrated that all investigated polymorphisms might result in altered gene expression or protein structure of CTLA-4 [16, 17]. Thus, it is likely that these polymorphisms might also influence normal functioning of CTLA-4, give rise to immune dysfunction, jeopardize anti-tumor immune responses, and influence predisposition to malignancies, and this is the reason why we investigated these polymorphisms in this meta- analysis. Second, although we pooled the results of related studies, the sample size of many comparisons were still relatively small, so future genetic association studies with larger sample sizes are still needed to estimate the relationship between CTLA-4 polymorphisms and different types of digestive system malignancies so as to get more statistically robust findings. Third, the etiologies and pathogenesis mechanisms of digestive system malignancies are extremely sophisticated, so further association studies also need to investigate the potential influence of gene-gene or geneenvironmental interactions on predisposition to digestive system malignancies [18]. Fourth, we aimed to investigate all CTLA-4 polymorphisms at the beginning. However, we did not find sufficient eligible articles to support pooled meta-analyses of other CTLA-4 polymorphisms, so we only examined six polymorphisms in this meta-analysis. 
Like all meta-analyses, a few limitations of our pooled meta-analyses should also be acknowledged. Firstly, our pooled meta-analysis results were derived from pooling unadjusted findings because we did not have access to raw data of eligible studies [19]. Secondly, environmental factors might also influence relationship between CTLA4 polymorphisms and digestive system malignancies. However, most investigators only focused on genetic associations in their works, so gene-environmental interactions were not explored in this meta-analysis [20]. Thirdly, we did not search for grey literatures. Therefore, despite that funnel plots of pooled analyses were overall symmetrical, potential publication biases still might influence the robustness of our pooled results [21].

\section{Conclusion}

So to conclude, this meta-analysis demonstrated that rs231775 polymorphism was associated with predisposition to colorectal cancer and pancreatic cancer, rs4553808 and rs5742909 polymorphisms were associated with predisposition to gastric cancer, rs3087243 polymorphism was associated with predisposition to liver cancer, and rs733618 polymorphism was associated with predisposition to colorectal cancer. These results indicated that these CTLA-4 polymorphisms might have the potential to serve as genetic biomarkers of digestive system malignancies. Nevertheless, detailed functional analyses are still required to reveal the precise molecular mechanisms of the observed significant associations between CTLA-4 polymorphisms and digestive system malignancies. Moreover, future studies should also test whether these CTLA-4 polymorphisms can be used to detect digestive system malignancies in clinical practice.

\section{Abbreviations}

Cl: Confidence intervals; CTLA-4: Cytotoxic T Iymphocyte-associated antigen 4; HWE: Hardy-Weinberg equilibrium; NOS: Newcastle-Ottawa scale; OR: Odds ratios

\section{Acknowledgements}

None.

\section{Authors' contributions}

$J L$ and $Y Z$ designed this study. $J$ and WW searched literatures. YS analyzed the data. $J \mathrm{~L}$ and $Y Z$ wrote the manuscript. All authors have approved the final manuscript as submitted.

\section{Funding}

None.

\section{Availability of data and materials}

The current study was based on the results of relevant published studies.

\section{Ethics approval and consent to participate}

Not applicable.

\section{Informed consent}

For this type of study formal consent is not required.

\section{Competing interests}

The authors declare that they have no competing interests.

\section{Author details}

${ }^{1}$ Department of Oncology, Changyi People's Hospital, Changyi 261300, Shandong Province, China. 'Department of Gastroenterology, Changyi People's Hospital, Changyi 261300, Shandong Province, China. ${ }^{3}$ Department of Gastroenterology, Zhuji Affiliated Hospital of Shaoxing University, Zhuji 311800, Zhejiang, China.

Received: 29 November 2019 Accepted: 26 January 2020

Published online: 16 March 2020

\section{References}

1. Siegel RL, Miller KD, Jemal A. Cancer statistics, 2017. CA Cancer J Clin. 2017; 67:7-30.

2. Ferlay J, Soerjomataram I, Dikshit R, Eser S, Mathers C, Rebelo M, Parkin DM, Forman D, Bray F. Cancer incidence and mortality worldwide: sources, methods and major patterns in GLOBOCAN 2012. Int J Cancer. 2015;136:E359-86.

3. Brenner H, Kloor M, Pox CP. Colorectal cancer. Lancet. 2014;383:1490-502.

4. Forner A, Llovet JM, Bruix J. Hepatocellular carcinoma. Lancet. 2012;379:1245-55.

5. Choi YJ, Kim N. Gastric cancer and family history. Korean J Intern Med. 2016; 31:1042-53.

6. Stenzinger A, Weichert W. Genetic profiling of cancers of the digestive system: biological insights and clinical implications. Pathobiology. 2017;84:306-22.

7. Sharma KL, Bhatia V, Agarwal P, Kumar A. Gastrointestinal cancers: molecular genetics and biomarkers. Can J Gastroenterol Hepatol. 2018;2018:4513860.

8. Stoffel EM. Screening in Gl cancers: the role of genetics. J Clin Oncol. 2015; 33:1721-8.

9. Chikuma S. CTLA-4, an essential immune-checkpoint for T-cell activation. Curr Top Microbiol Immunol. 2017:410:99-126.

10. Buchbinder El, Desai A. CTLA-4 and PD-1 pathways: similarities, differences, and implications of their inhibition. Am J Clin Oncol. 2016;39:98-106.

11. Moher D, Liberati A, Tetzlaff J, Altman DG, PRISMA group. Preferred reporting items for systematic reviews and meta-analyses: the PRISMA statement. Ann Intern Med. 2009;151:264-9.

12. Stang A. Critical evaluation of the Newcastle-Ottawa scale for the assessment of the quality of nonrandomized studies in meta-analyses. Eur J Epidemiol. 2010;25:603-5.

13. Paulsen EE, Kilvaer TK, Rakaee M, Richardsen E, Hald SM, Andersen S, Busund LT, Bremnes RM, Donnem T. CTLA-4 expression in the non-small cell lung cancer patient tumor microenvironment: diverging prognostic impact in primary tumors and lymph node metastases. Cancer Immunol Immunother. 2017;66:1449-61.

14. Wang SD, Li HY, Li BH, Xie T, Zhu T, Sun LL, Ren HY, Ye ZM. The role of CTLA-4 and PD-1 in anti-tumor immune response and their potential efficacy against osteosarcoma. Int Immunopharmacol. 2016;38:81-9.

15. Patel SS, Weirather JL, Lipschitz M, Lako A, Chen PH, Griffin GK, Armand P, Shipp MA, Rodig SJ. The microenvironmental niche in classic Hodgkin lymphoma is enriched for CTLA-4-positive T cells that are PD-1-negative. Blood. 2019;134:2059-69.

16. Gough SC, Walker LS, Sansom DM. CTLA4 gene polymorphism and autoimmunity. Immunol Rev. 2005;204:102-15.

17. Ghaderi A. CTLA4 gene variants in autoimmunity and cancer: a comparative review. Iran J Immunol. 2011;8:127-49.

18. Nishi A, Milner DA Jr, Giovannucci EL, Nishihara R, Tan AS, Kawachi I, Ogino S. Integration of molecular pathology, epidemiology and social science for global precision medicine. Expert Rev Mol Diagn. 2016;16:11-23.

19. Yuzhalin A. The role of interleukin DNA polymorphisms in gastric cancer. Hum Immunol. 2011;72:1128-36.

20. Min L, Chen D, Qu L, Shou C. Tumor necrosis factor-a polymorphisms and colorectal cancer risk: a meta-analysis. PLoS One. 2014;9:e85187.

21. Wu Z, Qin W, Zeng J, Huang C, Lu Y, Li S. Association between IL-4 polymorphisms and risk of liver disease: an updated meta-analysis. Medicine (Baltimore). 2015;94:e1435.

\section{Publisher's Note}

Springer Nature remains neutral with regard to jurisdictional claims in published maps and institutional affiliations. 